\title{
Autonomic and electrocardiographic findings in Parkinson's disease
}

\section{Christopher H. Gibbons ${ }^{\mathrm{a}, *}$ David K. Simon ${ }^{\mathrm{a}}$ Meilin Huang ${ }^{\mathrm{b}}$ Barbara Tilley $^{\mathrm{b}}$ Michael J. Aminoff $^{\mathrm{c}}$ Jacquelyn L. Bainbridge ${ }^{\mathrm{d}}$ Matthew Brodsky ${ }^{\mathrm{e}}$ Roy Freeman ${ }^{\mathrm{a}}$ John Goudreau $^{\mathrm{f}}$ Robert W. Hamill ${ }^{g}$ Sheng T. Luo ${ }^{\mathrm{b}}$ Carlos Singer ${ }^{\mathrm{h}}$ Aleksandar Videnovic ${ }^{\mathrm{i}}$ Ivan Bodis-Wollner $^{\mathrm{j}}$ Pei S. Wong ${ }^{\mathrm{k}}$ On behalf of theNINDS Exploratory Trials in Parkinson Disease (NET-PD) Investigators}

\footnotetext{
a Department of Neurology, Beth Israel Deaconess Medical Center and Harvard Medical School, 330 Brookline Avenue, Boston, MA 02215, USA

b Department of Biostatistics, University of Texas Health Science Center School of Public Health at Houston, Houston, TX 77030, USA

${ }^{c}$ Department of Neurology, School of Medicine, University of California, San Francisco, USA

d Department of Clinical Pharmacy and Neurology, University of Colorado, Anschutz Medical Campus, Skaggs School of Pharmacy and Pharmaceutical Sciences, Aurora, CO, USA

e Department of Neurology, Oregon Health \& Science University, USA

${ }^{\mathrm{f}}$ Department of Neurology, Michigan State University, USA

${ }^{g}$ Department of Neurological Sciences, University of Vermont College of Medicine, USA

h Department of Neurology, Miller School of Medicine, University of Miami, Miami, FL, USA

${ }^{i}$ Department of Neurology, Massachusetts General Hospital and Harvard Medical School, Boston, MA, USA

${ }^{j}$ Department of Neurology and Ophthalmology, State University of New York, Downstate Medical Center, USA

k Department of Pharmacy, Singapore General Hospital, Singapore
}

\section{A R T I C L E I N F O}

\section{Keywords:}

Parkinson's disease

Autonomic

Electrocardiogram

Heart rate variability

\begin{abstract}
A B S T R A C T
Parkinson disease (PD) is a progressive neurodegenerative disorder characterized by motor and non-motor symptoms and signs. Many reports suggest that diminished heart rate variability occurs early, even prior to the cardinal signs of PD. In a longitudinal study of PD, we evaluated whether heart rate variability (HRV) obtained using a 10-second ECG tracing, and the electrocardiographic QT-interval would be associated with PD severity and progression. Subjects were derived from a longitudinal study of 1741 individuals with early, stable PD. The severity of PD was measured using the global statistical test (GST). In a subset, the heart rate corrected QTinterval (QTcB) was calculated for each electrocardiogram (ECG). The HRV was measured for each ECG and then transformed to fit a normal distribution. The baseline analysis included 653 subjects, with 256 completing the 5year follow up study. There was an association $(P<0.05)$ between QTcB and PD severity in individuals that were taking QT-interval affecting drugs. A longer QT-interval at baseline was associated with more advanced PD at 5 years $(P<0.05)$, and greater disease progression over 5 years $(P<0.05)$. There was an association between diminished HRV and an orthostatic decrease in standing blood pressure at baseline in individuals with PD $(P<0.05)$. HRV was not associated with PD severity or progression. In conclusion, we were able to detect measurable associations between the QTcB interval and PD severity, PD severity 5 years later, and the change in disease over time. However, routine ECG tracings appear inadequate for the evaluation of autonomic function in PD.
\end{abstract}

\section{Introduction}

Parkinson disease (PD) is a progressive neurodegenerative disorder characterized by motor and non-motor symptoms and signs. Among the non-motor aspects of PD, autonomic dysfunction carries significant morbidity and is manifested by cardiovascular, gastrointestinal, urogenital, sudomotor, thermoregulatory or pupillary dysfunction (Arnao et al., 2015). As the incidence and prevalence of PD continues to grow in an aging population, the socioeconomic burden of autonomic dysfunction will continue to increase (Arnao et al., 2015; Cersosimo \& Benarroch, 2012).

Many reports identify cardiovascular autonomic dysfunction, characterized by diminished heart rate variability, in individuals with PD (Alonso et al., 2015; Bouhaddi et al., 2004; van Dijk et al., 1993). More recently, a large prospective study of individuals without PD was followed longitudinally and an association between diminished heart

\footnotetext{
* Corresponding author at: Autonomic and Peripheral Nerve Laboratory, Department of Neurology, Beth Israel Deaconess Medical Center, 1 Deaconess Road, Boston, MA 02215, USA.

E-mail address: cgibbons@bidmc.harvard.edu (C.H. Gibbons).
} 
rate variability and an increased risk of PD was detected (Alonso et al., 2015). In aggregate, these data suggest that diminished heart rate variability occurs early, even prior to the cardinal signs of PD. Furthermore, the peripheral degeneration of autonomic nerves in individuals with PD may predispose them to electrocardiographic prolongation of the QTc interval (Deguchi et al., 2002; Ishizaki et al., 1996; Oka et al., 1997). Finally, many classes of medications used to treat symptoms of PD may also prolong the QTc interval (Malek et al., 2013).

In the present study, we evaluated whether heart rate variability or the electrocardiographic QT interval, or both, are associated with the severity of PD and the rate of disease progression using routine ECG tracings. We hypothesized that individuals with more advanced Parkinson disease would have lower heart rate variability and longer corrected QT intervals on initial evaluation, and that these electrocardiographic abnormalities would correlate with greater overall severity and rate of progression of PD. We also hypothesized that longer QT interval and diminished heart rate variability, as markers of autonomic dysfunction, would be associated with orthostatic hypotension.

\section{Materials and methods}

\subsection{Subjects}

In 2001, the National Institute of Neurological Disorders and Stroke initiated the exploratory Trials of Parkinson Disease (NET-PD) program (Bega et al., 2015; Elm, 2012; Kieburtz et al., 2015; Parashos et al., 2014). A total of 1741 individuals with early, stable PD on dopaminergic therapy were enrolled (from March 2007 to May 2010) and studied longitudinally to test the hypothesis that creatine would alter disease progression (the NET-PD LS1 study) (Elm \& Investigators, 2012; Writing Group for the NETiPDI et al., 2015). The study was stopped due to futility based on an interim analysis after approximately half of the subjects had been in the study for 5 years (Writing Group for the NETiPDI et al., 2015). A subset of subjects had electrocardiograms (ECGs), including at baseline. Detailed examination scores, medications and questionnaires were obtained in all participating individuals. Orthostatic vital signs were recorded during the baseline and follow up visits from the supine and standing positions. Additional details of the trial have been published previously (Bega et al., 2015; Writing Group for the NETiPDI et al., 2015).

\subsection{Disease severity}

The severity of disease in the LS1 study was measured using the global statistical test (GST) as previously described (Bega et al., 2015). Briefly, the GST combines data from the following five outcome measures into a single quantifiable measure: the Modified Schwab and England activities of daily living (Louis et al., 1996), the Parkinson's Disease Quality of Life Scale (PDQ-39) (Jenkinson et al., 1997), a set of five questions from the United Parkinson Disease Rating Scale related to ambulatory capacity (2003), the Symbol Digit Modalities test (Smith, 2002), and the Modified Rankin (van Swieten et al., 1988). A higher GST scores indicates more advanced disease.

\subsection{Electrocardiogram}

Only individuals with a complete, good quality, 12-lead electrocardiograms at baseline were included. Good quality ECG's were defined as tracings with measureable electrocardiographic complexes without evidence of atrial fibrillation, other rhythm abnormalities, or a pacemaker. The heart rate corrected QT interval (QTcB) (Tsuji et al., 1996) was calculated for each ECG. The heart rate variability (HRV) was measured for each ECG tracing, reviewed to ensure biologic plausibility (i.e. that premature beats were not considered in the analysis), and then transformed logarithmically to fit a normal distribution.

\subsection{Analysis}

ECGs were performed at only a subset of centers participating in the LS1 study. We performed baseline comparisons between the demographic data for those LS1 study centers that performed ECGs, and those that did not. Within each center that did perform ECGs we compared the demographic data between those individuals that had ECGs and those that did not. Finally we compared the demographic data of those individuals with good quality ECG tracings to those with low quality tracings that were excluded.

Associations between baseline QT Interval with baseline GST, 5-year GST, and GST change from baseline to 5 years were examined. Also, the association between baseline heart rate variability and baseline GST, 5year GST, and GST change from baseline to 5-year point was assessed. Similarly, association of baseline QT Interval with baseline orthostatic change in blood pressure (defined as change in systolic BP from the supine to standing position), 5-year orthostatic change in BP, and orthostatic change in BP from baseline to 5-year level, association of baseline heart rate variability with baseline orthostatic change in BP, 5year orthostatic change in $\mathrm{BP}$, and orthostatic change in BP from baseline to 5-year point were studied.

Mixed model was used for the data analysis. The statistical analysis controlled for age, duration since PD diagnosis, gender, PD medication (dopamine agonist, levodopa, or both), PD medication dose (levodopa equivalent baseline dose), comorbidity score at baseline, and the number of QTcB length-affecting drugs (taken for least a week before the baseline ECG). Treatment group and site, a stratifying variable, were two random effects in the model. Interactions were treated as significant at the 0.1 level. Subgroup analyses were conducted when interactions were detected. In this analysis we only adjusted for QTcB length-affecting drugs that increased QTcB length. We did not have any information on the direction of the heart rate variability drug effect (e.g. increasing or decreasing HRV). When there was an effect of age in the model, the groups were divided into approximate quartiles of the range for additional analysis (age $<57,57 \leq$ age $<62,62 \leq$ age $<$ 67 , or age $\geq 67$ ).

PD medication was treated as three dummy variables (levodopa only, dopamine agonist only, and both where "both" is the reference group). For the purposes of this analysis, dopaminergic therapy was defined as either levodopa or a dopamine agonist, as this was part of the study inclusion criteria. Other indirect dopaminergic therapies (such as monoamine oxidase inhibitors) were included the overall analysis of drugs that could impact QTcB or HRV. The number of QTcB length affecting drugs being taken at baseline was treated as one binary variable (number of QTcB length affecting drugs $=0$ or $>0$, where " 0 " is the reference group), and the number of HRV affecting drugs being taken at baseline was also treated as one binary variable (number of affecting drugs $\leq 1$ or $\geq 2$, where " $\leq 1$ " is the reference group).

\section{Results}

Technically interpretable ECG tracings, physical examination scores, and demographic data (Table 1) were available for analysis in 653 subjects at the baseline visit, with 256 of those subjects completing the 5-year follow up study. The distribution and exclusion of all subjects is shown in Fig. 1. Subjects from centers that performed ECGs tended to have a longer duration of parkinsonian symptoms, a higher levodopa equivalent baseline dose usage, and a lower ambulatory capacity score than subjects from the centers that did not perform ECGs. Within centers that performed ECGs, male gender, duration of PD symptoms, levodopa equivalent baseline dose, Schwab \& England scale, symbol digit modalities, and modified Rankin scores are all significantly different for subjects who had an ECG compared to those who did not 
Table 1

Demographic data of study subjects.

\begin{tabular}{|c|c|c|c|c|}
\hline Baseline variable & $\begin{array}{l}\text { ECG center } \\
(N=1027)\end{array}$ & $\begin{array}{l}\text { No ECG center } \\
(N=714)\end{array}$ & $\begin{array}{l}\text { Included subjects } \\
(N=653)\end{array}$ & Excluded subjects $(N=161)$ \\
\hline Age (mean years) ${ }^{\mathrm{a}}$ & 61.82 & 61.75 & 61.1 & $63.8^{\dagger}$ \\
\hline Male (frequency $\%)^{b}$ & 65.14 & 63.59 & 64.2 & $61.5^{\dagger}$ \\
\hline \multicolumn{5}{|l|}{ PD characteristics } \\
\hline Time since PD Diagnosis (yrs) ${ }^{\mathrm{a}}$ & 1.58 & 1.49 & 1.56 & 1.66 \\
\hline Duration of PD symptoms (yrs) ${ }^{\mathrm{a}}$ & 3.37 & $3.13^{*}$ & 3.28 & $3.71^{\dagger}$ \\
\hline Total daily LEDD $(\mathrm{mg})^{\mathrm{a}}$ & 389.44 & $373.49^{*}$ & 378.1 & $360.1^{\dagger}$ \\
\hline Schwab \& England Activities of daily living ${ }^{\text {a }}$ & 91.06 & 91.18 & 91.5 & $90.9^{\dagger}$ \\
\hline PDQ-39 summary index ${ }^{a}$ & 13.13 & 13.41 & 12.9 & 13.2 \\
\hline Symbol digit modalities test ${ }^{\mathrm{a}}$ & 44.86 & 43.87 & 45.9 & $43.0^{\dagger}$ \\
\hline Ambulatory capacity ${ }^{\mathrm{a}}$ & 1.65 & $1.78^{*}$ & 1.57 & 1.83 \\
\hline Modified ranking score ${ }^{a}$ & 1.20 & 1.25 & 1.17 & $1.20^{\dagger}$ \\
\hline Comorbidity $(\% \text { with }>0)^{\mathrm{b}}$ & 91.04 & 88.10 & 90.51 & 95.03 \\
\hline
\end{tabular}

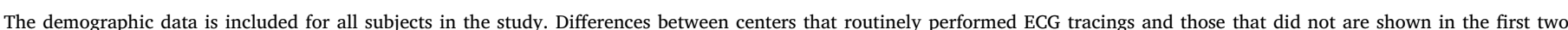

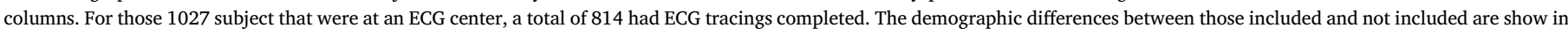

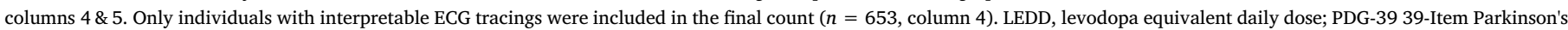
Disease Questionnaire; UPDRS, Unified Parkinson Disease Rating Scale.

${ }^{*} P<0.05$ vs. ECG center.

${ }^{\dagger} P<0.05$ vs. included subjects.

${ }^{a}$ Wilcoxon rank-sum test was used.

b Chi-square test was used. Given the multiple tests conducted, the statistically significant results in Table 1 could have happened by chance alone.

have an ECG at baseline. Among those individuals who had an ECG, age, duration of PD symptoms, baseline comorbidity scores, and baseline symbol digit modalities differed for those with good quality tracings compared to those whose electrocardiogram tracings were not included. Given the number of tests conducted, none of these differences achieve statistical significant if corrected for multiple comparisons.

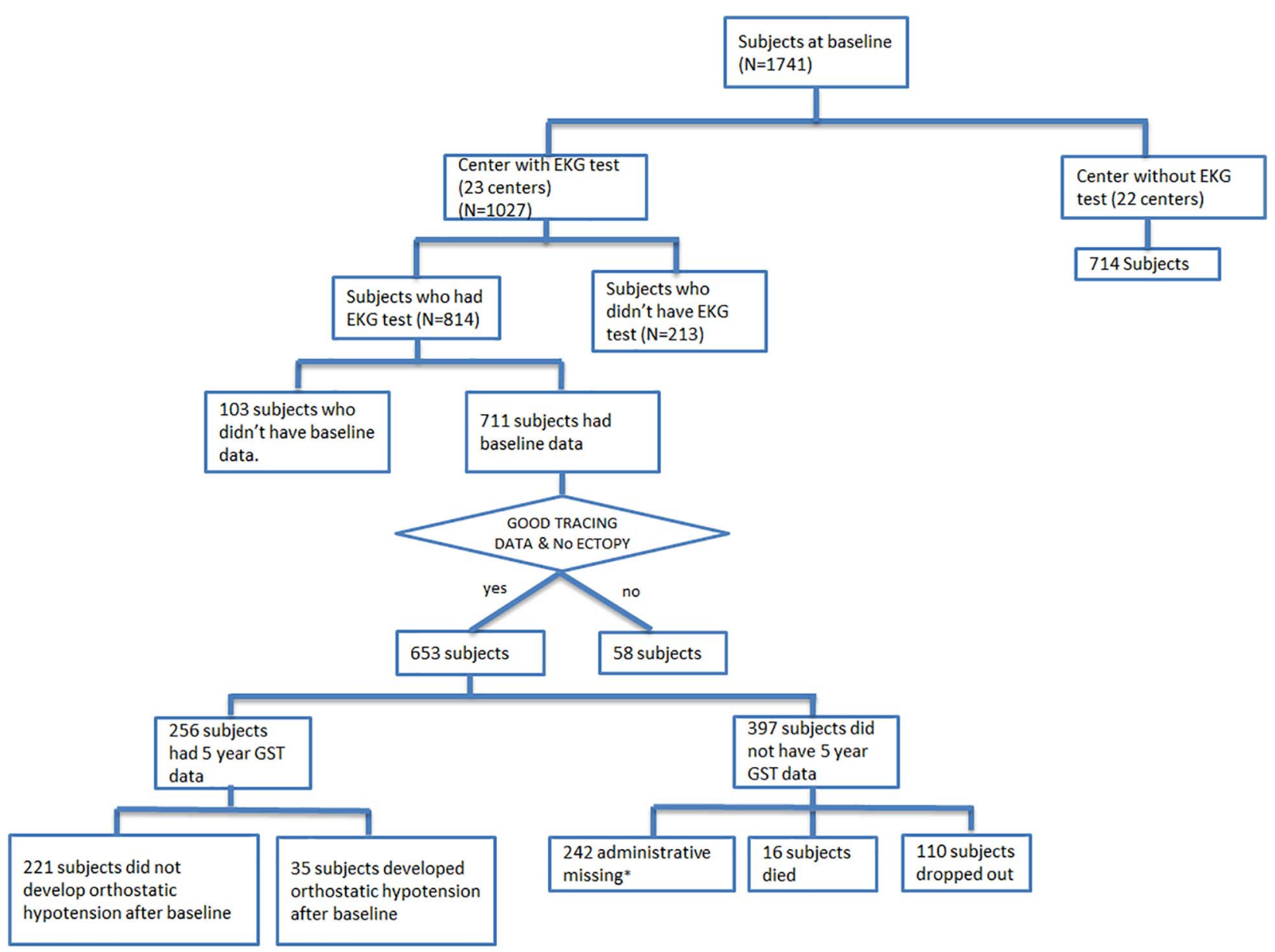

*Administrative missing: Study ended before subjects could reach five years.

Fig. 1. The distribution of the 1741 LS-1 subjects across the ECG study. 


\subsection{QT interval at baseline}

In the model, there was an interaction between the QTcB interval and the number of drugs that affect the QT interval taken at baseline. The data were therefore categorized into participants who took drugs that affected the QT interval and those who did not (with the exception of levodopa or dopamine agonists: all subjects were taking one or more of these medications). The levodopa equivalent dose and comorbidity score at baseline were significantly (all $P<0.05$ ) associated with the baseline GST for participants independent of whether they took QT interval affecting drugs at baseline. However, the QTcB interval at baseline was only significantly associated with baseline GST for subjects who took QT interval affecting drugs at baseline. Thus, among the subjects who took QT interval affecting drugs at their baseline visit, a higher QTcB was associated with a higher GST score.

In the evaluation of QT interval at baseline and baseline orthostatic change in BP, there was an interaction between age and baseline corrected QT interval in the model. Data were categorized into four groups based on age: age $<57,57 \leq$ age $<62,62 \leq$ age $<67$, or age $\geq 67$. In the subgroups where subjects age is $\leq 62$, baseline corrected QT interval was associated with baseline orthostatic change in BP $(P=0.05)$ thus those individuals with longer corrected QT intervals had a greater degree of orthostatic hypotension.

\subsection{QT interval and 5-year follow up}

There was an interaction between age and corrected QT interval length in the model. Data were categorized based on the quartile groups by age. Baseline corrected QT interval length was associated with 5year GST score for subjects over 67 years old $(P<0.05)$; thus individuals over the age of 67 with a longer corrected QT interval will have higher 5-year GST scores.

\subsection{QT interval and change in 5-year GST score}

There was an interaction between age and baseline corrected QT interval in the model. Data were categorized based on the quartile groups by age. In the model, baseline corrected QT intervals are significantly associated with GST score changes from baseline to 5-year for subjects over 67 years of age. Similar to the results for QT interval and 5-year follow up, a longer baseline corrected QT interval will be associated with a greater increase in GST score over 5 years for subjects older than 67 years.

\subsection{Heart rate variability at baseline}

There was a three-way interaction between baseline HRV, comorbidity score, and levodopa equivalent dose in the model. An association between numbers of drugs affecting heart rate interval taken at baseline with baseline GST could not be detected. We categorized the data based on the baseline comorbidity score of 0 or $>0$. The levodopa equivalent dose and duration of PD medication was associated with the baseline heart rate variability in subjects whose comorbidity scores were $>0$ $(P<0.05)$. There was no relationship between HRV, levodopa equivalent dose, type of PD medication and baseline GST score in subjects whose baseline comorbidity score was zero. Thus, at baseline, both age and HRV were significantly associated with orthostatic change in BP (i.e. greater age or lower HRV was associated with greater orthostatic change in $\mathrm{BP}$ ).

\subsection{Heart rate variability and 5-year follow up}

There was an interaction between age and the log-transformed HRV in the model. Data were categorized based on the quartile groups by age. In the model, an association between the baseline HRV and the 5year GST score could not be detected. In subgroups where age is $<57$ years, there is an association between HRV and the 5-year GST scores where lower baseline HRV was associated with greater 5-year GST scores $(P<0.05)$.

\subsection{Heart rate variability and change in 5-year GST score}

There was an interaction between age and baseline HRV in the model. Data were categorized based on the quartile groups by age. Similar to the findings with baseline HRV and the 5-year GST score, there was an inverse association between baseline HRV and GST score change at 5 years for subjects aged $<57$ years $(P<0.05)$. Thus individuals with low HRV at baseline tended to have greater GST score and greater change in GST scores at 5-years.

\section{Discussion}

This is a large longitudinal investigation of the association between HRV, QT interval, and PD. We report several novel findings in this study. First, we found a significant positive association between the corrected QT interval and PD severity (as measured by the Global Statistical Test) in individuals taking drugs that affect the QT interval, particularly if they are over the age of 67. Second, a longer QT interval at baseline was associated with more advanced disease at 5 years, and greater disease progression over 5 years. There was no association between HRV and PD severity or progression, although we suspect that study limitations (including selective bias and the inadequacy of routine ECG data) may have prevented our ability to detect this potential interaction. Finally, we detected an association between diminished HRV and baseline orthostatic change in BP in individuals with PD.

The finding of an association of corrected QT interval with PD severity in patients taking at least one drug affecting QT interval, but not in patients not taking such drugs, is interesting. One possible explanation is that, in this early PD population, spontaneous QT prolongation is relatively infrequent, but a predisposition to QTprolongation is "unmasked" by a drug that affects QT-intervals, thereby making it possible to detect the association with greater PD severity. It should also be noted that the clinical significance of these QT interval changes is not clear as most values were within the normative range.

Several population-based studies of cardiovascular autonomic neuropathy and PD exist (Alonso et al., 2015; Palma et al., 2013; van Dijk et al., 1993). However, due to the relatively small number of people with PD in the general population, many population based studies include only a small number of people with PD and conclude that a larger longitudinal study is required (Alonso et al., 2015; Palma et al., 2013; van Dijk et al., 1993). Large-scale studies of autonomic function in PD are lacking, partly because detailed autonomic assessment of cardiovascular function requires prolonged HRV recording and more complex tests of autonomic function. Inevitably, these autonomic assessments are left out of large clinical trials where the additional expense and logistical challenges outweigh the perceived benefits. Recognizing these limitations, we wanted to test the hypothesis that a standard 12-lead ECG could provide sufficient data for analysis of cardiovascular autonomic function in a large study of individuals with PD.

The current study is consistent with previous reports that patients with PD often take many medications that may prolong the QT interval (Cunnington et al., 2013; Malek et al., 2013). Approximately one-third of our study cohort was taking at least 1 drug that altered QT interval. Other investigators have advocated for routine ECGs in individuals with PD who are taking medications that affect the QT interval (Cunnington et al., 2013). In the present study, among those testing centers that performed ECGs, those individuals that did not receive an ECG on average were older, had PD for a longer duration, had greater disability scores at baseline and took greater levodopa equivalent doses at baseline. One could imagine that it may be more difficult to obtain 
ECGs in those individuals with more severe disease or disability, but these more advanced PD patients may be precisely the ones who could benefit the most from a screening ECG. The relationship between disease severity or disability and clinically important ECG changes should factor into the clinical decision to pursue this additional diagnostic test in the context of physical challenges and time constraints during a clinical evaluation and may highlight a subtle bias against testing in some situations. Additional study and clinical follow up may be necessary to understand the impact of this problem.

We also extracted the HRV from our 10-second ECG recordings. Other investigators have used routine ECG analysis of heart rate in large-scale studies to study medication effects (Noordam et al., 2015) or disease severity or progression (Lykke et al., 2008). There were sufficient numbers of individuals with some degree of HRV in our study that we could detect an association between reduced HRV and orthostatic change in BP. As expected, those individuals with more pronounced postural change in blood pressure had very low HRV. However, we did not detect an association between HRV and PD severity or PD progression. This information contrasts with prior studies that have established a connection between a decline in HRV and progression of PD (Alonso et al., 2015; van Dijk et al., 1993). We surmise that the lack of association between HRV and PD severity or PD progression in the present study is simply due to inadequate data acquisition. The majority of individuals in our cohort had $<2$ beats per minute of variability detectable within the sampling region, an unexpected finding for PD patients without severe autonomic dysfunction (Alonso et al., 2015). Our interpretation of these data is that the 10-second ECG does not provide sufficient sampling to avoid a 'floor' effect. Therefore, we conclude that the routine ECG is inadequate for assessment of HRV in individual subjects or small patient groups with PD. The findings of an association between HRV and orthostatic hypotension, and an association between the QT interval and the number of drugs taken that affect the QT interval are expected. Many prior studies have documented similar findings. However, the ability to detect these changes using routine ECG tracings does suggest that some limited amount of information may be garnered from studies with sufficient numbers of patients. However, investigators should be extremely cautious in the interpretation of HRV data using routine ECG tracings because the data is not a surrogate for properly performed heart rate variability testing.

The present study has a number of limitations. This was a retrospective analysis of data gathered during a clinical trial. The analysis was exploratory, and $P$-values for subgroup analyses were not adjusted for multiple comparisons. If corrections for multiple comparisons were included, most of the findings would not have been statistically significant. However, because subgroup comparisons were made only when interactions were detected, a weak protection of alpha was provided to the analysis. The data acquired were restricted to only a subset of the study participants, and selective bias against more advanced disease may have skewed the results. Finally, it appears that the use of routine 10-second ECG tracings is not sufficient to adequately investigate HRV.

\section{Conclusions}

We were able to detect measurable associations between the corrected QT interval and PD severity, the disease severity 5 years later, and the change in disease over time. However, we were unable to detect associations between HRV and PD severity or PD progression. These findings suggest that $10 \mathrm{~s}$ ECG tracings are inadequate for the evaluation of autonomic function in PD and its relationship to disease severity and progression.

\section{Authors' roles}

Project Conception: Simon, Project Execution: Gibbons, Simon,
Huang, Tilley, Aminoff, Bainbridge, Brodsky, Freeman, Goudreau, Hamill, Luo, Singer, Videnovic, Bodis-Wollner, Wong. Statistical Analysis: Huang, Tilley. Statistical Review: Gibbons, Simon. Writing of first draft: Gibbons. Review and Critique: Gibbons, Simon, Huang, Tilley, Aminoff, Bainbridge, Brodsky, Freeman, Goudreau, Hamill, Luo, Singer, Videnovic, Bodis-Wollner, Wong.

\section{Funding sources for study}

Financial support for the LS-1 study was provided by National Institute of Neurological Disorders and Stroke (NINDS) grant U01NS43128.

\section{Financial disclosure/conflict of Interest}

None relevant to this research. Full disclosures provided at the end of the manuscript.

\section{References}

Alonso, A., Huang, X., Mosley, T.H., Heiss, G., Chen, H., 2015. Heart rate variability and the risk of Parkinson disease: the atherosclerosis risk in communities study. Ann. Neurol. 77 (5), 877-883.

Arnao, V., Cinturino, A., Valentino, F., Perini, V., Mastrilli, S., Bellavia, G., Savettieri, G., Realmuto, S., D'Amelio M., 2015. In patient's with Parkinson disease, autonomic symptoms are frequent and associated with other non-motor symptoms. Clin. Auton. Res. 25 (5), 301-307 (Oct).

Bega, D., Kim, S., Zhang, Y., Elm, J., Schneider, J., Hauser, R., et al., 2015. Predictors of functional decline in early Parkinson's disease: NET-PD LS1 cohort. J. Park. Dis. 5 (4), 773-782.

Bouhaddi, M., Vuillier, F., Fortrat, J.O., Cappelle, S., Henriet, M.T., Rumbach, L., et al., 2004. Impaired cardiovascular autonomic control in newly and long-term-treated patients with Parkinson's disease: Involvement of L-dopa therapy. Auton. Neurosci. 116 (1-2), 30-38.

Cersosimo, M.G., Benarroch, E.E., 2012. Autonomic involvement in Parkinson's disease: pathology, pathophysiology, clinical features and possible peripheral biomarkers. J. Neurol. Sci. 313 (1-2), 57-63.

Cunnington, A.L., Hood, K., White, L., 2013. Outcomes of screening Parkinson's patients for QTc prolongation. Parkinsonism Relat. Disord. 19 (11), 1000-1003.

Deguchi, K., Sasaki, I., Tsukaguchi, M., Kamoda, M., Touge, T., Takeuchi, H., et al., 2002 Abnormalities of rate-corrected QT intervals in Parkinson's disease-a comparison with multiple system atrophy and progressive supranuclear palsy. J. Neurol. Sci. 199 (1-2), 31-37.

van Dijk, J.G., Haan, J., Zwinderman, K., Kremer, B., van Hilten, B.J., Roos, R.A., 1993. Autonomic nervous system dysfunction in Parkinson's disease: relationships with age, medication, duration, and severity. J. Neurol. Neurosurg. Psychiatry 56 (10), 1090-1095.

Elm, J.J., 2012. Design innovations and baseline findings in a long-term Parkinson's trial: the national Institute of neurological disorders and stroke exploratory trials in Parkinson's disease long-term study-1. Mov. Disord. 27 (12), 1513-1521.

Elm, J.J., Investigators, N.N.-P., 2012. Design innovations and baseline findings in a longterm Parkinson's trial: the national institute of neurological disorders and stroke exploratory trials in Parkinson's disease long-term study-1. Mov. Disord. 27 (12), 1513-1521.

Ishizaki, F., Harada, T., Yoshinaga, H., Nakayama, T., Yamamura, Y., Nakamura, S., et al., 1996. No to Shinkei 48 (5), 443-448 (Brain and nerve).

Jenkinson, C., Fitzpatrick, R., Peto, V., Greenhall, R., Hyman, N., 1997. The Parkinson's disease questionnaire (PDQ-39): development and validation of a Parkinson's disease summary index score. Age Ageing 26 (5), 353-357.

Kieburtz, K., Tilley, B.C., Elm, J.J., Babcock, D., Hauser, R., Ross, G.W., et al., 2015. Effect of creatine monohydrate on clinical progression in patients with Parkinson disease: a randomized clinical trial. JAMA 313 (6), 584-593.

Louis, E.D., Lynch, T., Marder, K., Fahn, S., 1996. Reliability of patient completion of the historical section of the Unified Parkinson's Disease Rating Scale. Mov. Disord. 11 (2), 185-192 (Mar, PubMed PMID: 8684390).

Lykke, J.A., Tarnow, L., Parving, H.H., Hilsted, J., 2008. A combined abnormality in heart rate variation and QT corrected interval is a strong predictor of cardiovascular death in type 1 diabetes. Scand. J. Clin. Lab. Invest. 1-6.

Malek, N.M., Grosset, K.A., Stewart, D., Macphee, G.J., Grosset, D.G., 2013. Prescription of drugs with potential adverse effects on cardiac conduction in Parkinson's disease. Parkinsonism Relat. Disord. 19 (6), 586-589.

Movement Disorder Society Task Force on Rating Scales for Parkinson's Disease, 2003. The Unified Parkinson's Disease Rating Scale (UPDRS): status and recommendations. Mov. Disord. 18 (7), 738-750 (Jul, Review. PubMed PMID: 12815652).

Noordam, R., van den Berg, M.E., Niemeijer, M.N., Aarts, N., Hofman, A., Tiemeier, H., et al., 2015. Antidepressants and heart-rate variability in older adults: a populationbased study. Psychol. Med. 1-9.

Oka, H., Mochio, S., Sato, H., Katayama, K., 1997. Prolongation of QTc interval in patients with Parkinson's disease. Eur. Neurol. 37 (3), 186-189.

Palma, J.A., Carmona-Abellan, M.M., Barriobero, N., Trevino-Peinado, C., Garcia-Lopez 
M., Fernandez-Jarne, E., et al., 2013. Is cardiac function impaired in premotor Parkinson's disease? A retrospective cohort study. Mov. Disord. 28 (5), 591-596. Parashos, S.A., Luo, S., Biglan, K.M., Bodis-Wollner, I., He, B., Liang, G.S., et al., 2014

Measuring disease progression in early Parkinson disease: the national institutes of health exploratory trials in Parkinson disease (NET-PD) experience. JAMA Neurol. 71 (6), 710-716.

Smith, A., 2002. Symbol Digit Modalities Test Manual. Los Angeles, CA, Western Psychological Services. van Swieten, J.C., Koudstaal, P.J., Visser, M.C., Schouten, H.J., van Gijn, J., 1988.

Interobserver agreement for the assessment of handicap in stroke patients. Stroke 19 (5), 604-607.

Tsuji, H., Venditti Jr., F.J., Manders, E.S., Evans, J.C., Larson, M.G., Feldman, C.L., et al., 1996. Determinants of heart rate variability. J. Am. Coll. Cardiol. 28 (6), 1539-1546. Writing Group for the NETiPDI, Kieburtz, K., Tilley, B.C., Elm, J.J., Babcock, D., Hauser, R., et al., 2015. Effect of creatine monohydrate on clinical progression in patients with Parkinson disease: a randomized clinical trial. JAMA 313 (6), 584-593. 